\title{
An experimental setup to measure the heat-exchange processes by controlling thermal and hydraulic conditions
}

\author{
Paolo Scotton \\ Giorgia Dalla Santa
}

\author{
Giordano Teza \\ Antonio Galgaro
}

\author{
Daniele Rossi
}

\begin{abstract}
The design of a Borehole Heat Exchanger (BHE) is based on the evaluation of the thermal exchange capacity of the whole system constituted by the probes and the surrounding ground. The energy performance of a BHE mainly depends on the thermal properties of the sediments, the possible groundwater flow and the changes in the thermal gradient in the probe's surroundings due to the continuous heat exchange with the subsoil. The interpretation of the in-field applications is often difficult because in many instances the information needed is unavailable due to difficulties of in-field measurements. An experimental device was built in order to assess, under controlled conditions, the evolution in time and space of the energetic processes that occur between a thermal probe and the surrounding ground. A copper probe was placed into a soil control volume of $1 \mathrm{~m}^{3}$ and 24 bigh precision temperature sensors were distributed inside this volume at different distances from the probe. The configuration of the experimental settings was built to allow alterations in terms of sediments, groundwater flow conditions, thermal probe properties and operations, in order to simulate different physical conditions and to better understand the complex physical processes involved. Another goal of the experimental research was to produce reliable experimental data that can be used for the calibration and set up of numerical models. This paper describes the experimental apparatus and two experiments performed in order to assess its capability to satisfy the design requirements.
\end{abstract}

\section{INTRODUCTION}

The physical phenomena that occur in a borehole heat exchanger (BHE) are generally well known and welldefined technical solutions are currently available thanks to the achievements obtained worldwide in the last 20-30 years in terms of design, installation and use of ground coupled heat pumps (GCHPs). However, there are still some open issues. The design of a BHE to be used in a shallow geothermal system is based on the evaluation of the thermal exchange capacity of the whole system constituted by the BHE itself and the surrounding ground. The energy performance of the system mainly depends on the thermal properties of the sediments and on possible groundwater flow (Sutton 2003). Moreover, the continuous heat exchange with the subsoil modifies the thermal status of the subsoil itself which, in turn, affects the thermal gradient in the BHE surroundings. The approach to BHE modeling can be analytical (see e.g. Philippe et al. 2009) or numerical (see e.g. Al-Khoury et al. 2005; Al-Khoury and Bonnier 2006). Examples of modeling focusing on district heating, i.e. teleheating, also exist (Carlini et al. 2012). Regardless to the used approach, the thermal alteration induced in the ground and the BHE performance are usually evaluated by means of models of heat transfer and groundwater flow that need to be validated through the comparison with experimental data, which are often missing due to the difficulties of in-field measurements. Therefore, in some cases

Paolo Scotton (paolo.scotton@unipd.it), Giordano Teza (giordano.teza@unipd.it), Giorgia Dalla Santa (giorgia.dallsanta@unipd.it) and Antonio Galgaro (anotonio.galgaro@unipd.it) perform their reserach activities at the Department of Geosciences, University of Padua, Italy. Daniele Rossi (danielerossi74@gmail.com) is a consultant engineer. 
the design of a BHE may not be enough accurate and an assessment of its environmental and economic benefits could be inadequate.

Physical models are valid tools for the solution of many natural phenomena that are difficult to solve analytically or numerically as initial value problems or boundary value problems, or whose solution obtained with these methods has some uncertainties (Green 2014; Volkov et al. 2017). This tools are often used to verify the results of an analytical or numerical application obtained through appropriate simplifications. Physical modeling can also be used to describe the complex hydro-thermodynamic interaction that occurs when a thermal probe exchanges energy with the surrounding environment. For example, Wang et al. (2013) built a $900 \times 800 \mathrm{~mm}$ experimental setup in order to investigate the interactions between soil and pipes in case of ground freezing phenomena: they analyzed the effects of soils with different grain sizes on the movement of the freezing front and on the deformations induced on the pipes. Cimmino et Bernier (2015) built an experimental setup constituted of a small-scale $400 \mathrm{~mm}$-long borehole inserted into a $2 \mathrm{~m}^{3}$ sand tank with known thermal properties in order to obtain the experimental g-function, starting from measurements of the borehole wall temperature and of the net heat injection. Subsequently, the experimental gfunction was compared to the g-function obtained analytically. Recently, Li et al (2018) built a $6.25 \mathrm{~m} \times 1.5 \mathrm{~m} \times 1 \mathrm{~m}$ experimental box to investigate the transient heat transfer performance of a double-U probe in a layered ground, then comparing the results with the outputs of a 3D numerical model.

A physical model of a probe-ground system was built (Scotton 2017) in order to measure, under controlled conditions, the evolution in time and space of the thermal alteration induced in the ground by a thermal probe. It was a single probe, inserted into a volume of $1 \mathrm{~m}^{3}$, where 24 high precision temperature sensors were distributed at different distances from the probe. The experimental settings could be varied in a wide range of configurations in terms of sediments (with their different porosity, thermal properties, and hydraulic permeability), groundwater flow conditions and thermal probe operations. In order to better understand the complex physical processes involved, in the present early stages of the experimentations the thermal exchange was magnified by using a copper probe instead of a PE probe, obtaining a thermal power per unit length higher than the typical one. This paper shows details about the experimental setup and preliminary experimental results.

\section{THE EXPERIMENTAL APPARATUS}

\section{Supporting structure}

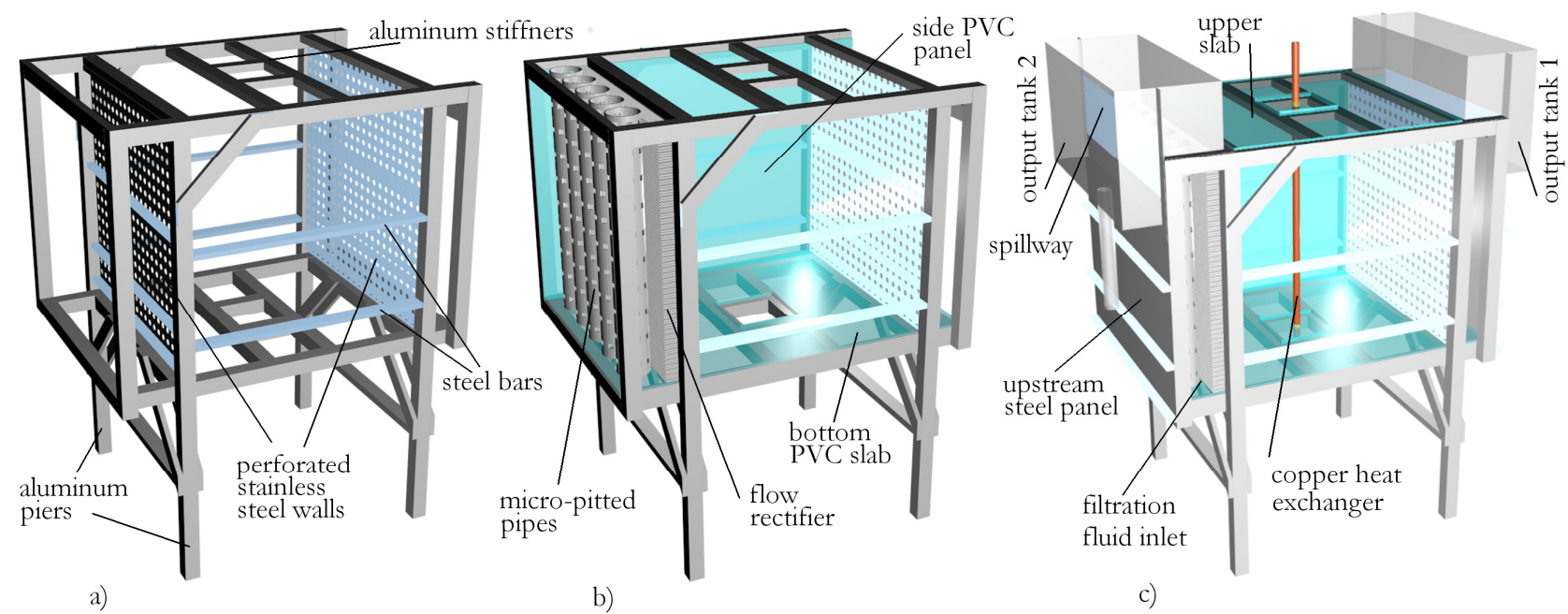

Figure 1 The bearing structure of the experimental apparatus. The materials used are aluminum, steel and PVC.

The supporting structure of the experimental apparatus is shown in Figure 1. It has a height of approximately 2 $\mathrm{m}$, a length of $1.5 \mathrm{~m}$, a width of $1.1 \mathrm{~m}$ and a mass of $200 \mathrm{~kg}$. The maximum weight of the experimental device, in test 
configuration, is $3000 \mathrm{~kg}$. The load-bearing structure is made of aluminum. The aluminum piers have a square section of $6 \mathrm{~cm}$ side. The supporting elements of the side walls, the steel bars, have a rectangular section of $6 \mathrm{~cm} \times 2 \mathrm{~cm}$.

The internal support of the granular material is obtained using $2-\mathrm{mm}$ thick perforated stainless steel walls (Figure 1.a). The area of the holes, which accounts for approximately $50 \%$ of the total surface, allows the free passage of the filtration fluid, inlet and outlet from the porous medium. The bottom of the apparatus is made of a $20 \mathrm{~mm}$ thick opaque PVC slab. The side walls are made of $10 \mathrm{~mm}$ thick transparent PVC panels. Figure 1.b shows the distributors of the filtration fluid, made with micro-pitted PVC pipes, and the flow rectifiers, made of polycarbonate honeycomb sheets. Figure 1.c displays the upstream and downstream closure steel walls of the model and the output tanks of the filtration fluid. The two tanks are equipped with spillways to stabilize the piezometric elevation of the filtration fluid in and out of the model. The maximum piezometric gradient obtainable is about $0.25 \mathrm{~m} / \mathrm{m}$. Figure 1.c also shows the copper pipe, used as a heat exchanger in the experimental tests reported in this article. The copper pipe was chosen to enhance the effects of the energy exchange between the probe and the surrounding environment. The internal diameter of the pipe is $28 \mathrm{~mm}$, the thickness is $2 \mathrm{~mm}$, the length is $0.95 \mathrm{~m}$. The upper closing plate is a $1 \mathrm{~cm}$ thick transparent PVC slab.

Figure 2 shows the physical model carried out according to the design. The aluminum piers rest on heightadjustable supports which allow to level the structure.
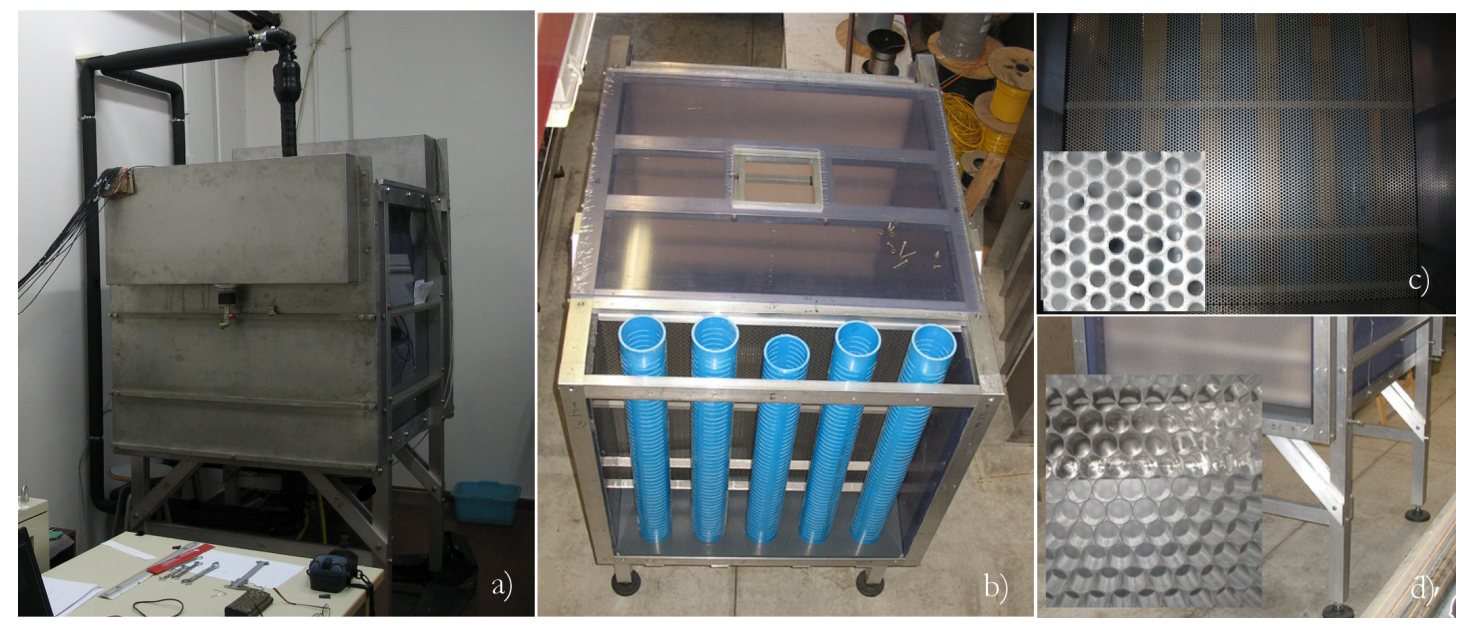

Figure 2 a) The experimental apparatus in test configuration. The insulated circuit of the copper heat exchanger is visible; b) A view from the top of the filtration fluid inlet tank with the micro-pitted pipes; c) The perforated stainless steel wall; d) A detail of the flow rectifier located between the micro-pitted pipes and the perforated wall.

\section{Hydraulic circuits}

The physical apparatus described above has two hydraulic circuits: the hydraulic circuit of the cold fluid (HCC) and the hydraulic circuit of the hot fluid $(\mathrm{HCH})$. Both circuits can be plugged into the probe circuit or into the filtration fluid circuit. The structural part of the experimental device and the connection points of the circuit of the thermal probe and of the filtration fluid are schematized in Figure 3, on the left. The probe circuit inserts the fluid into the upper part of the copper probe (inputCS) and reacquires it at the lower end of the probe (outputCS). The filtration fluid circuit inserts the fluid into the lower part of the loading tank (inputCF). The stability of the filtration motion through the porous medium of the experimental device is ensured by means of two spillways, placed in the loading tank and in the discharge tank respectively. Therefore, there are two discharges of the filtration circuit: one from the discharge tank (outputCF1) and one from the loading tank (outputCF2). The two circuits are designed to ensure a steady flow rate with constant temperature at the model inputs. 

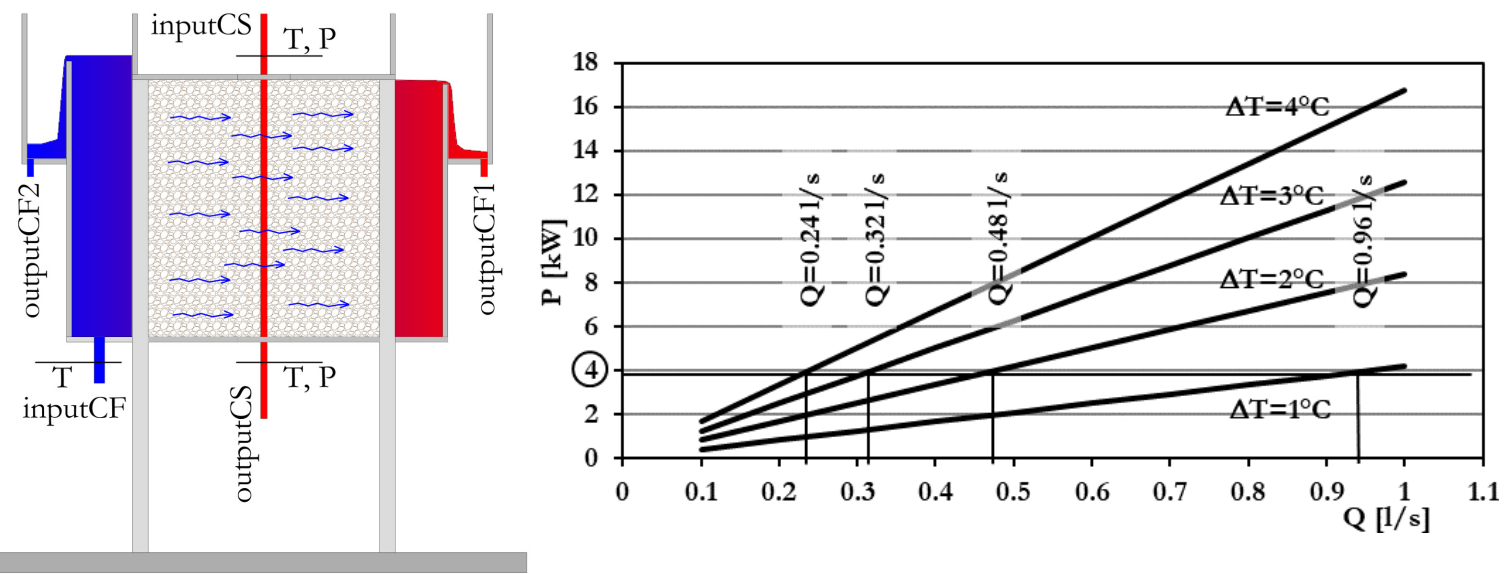

Figure 3 On the left, a scheme of the experimental apparatus with indication of input and output points of the cold and hot hydraulic circuit; on the right, power of the thermal machine vs flow rate when the temperature in the probe circuit fluid (water) varies between $1^{\circ} \mathrm{C}$ and $4^{\circ} \mathrm{C}$.

Thermal power, flow rate and flow regime in the hydraulic circuits. The flow rate in the hydraulic circuits is a function of the temperature variation that the fluids undergo by covering the entire circuit. This temperature difference, in heating or cooling, must be restored by a machine of adequate power. Figure 3, on the right, shows the dependence between the power of the thermal machine and the flow rate that can be managed when the assumed temperature variation in the circuit varies between $1^{\circ} \mathrm{C}$ and $4^{\circ} \mathrm{C}$. If a $4 \mathrm{~kW}$ power machine is used, the flow rate varies between $0.24 \mathrm{l} / \mathrm{s}\left(4^{\circ} \mathrm{C}\right.$ temperature variation in the circuit) and $0.961 / \mathrm{s}\left(1^{\circ} \mathrm{C}\right.$ temperature variation in the circuit).
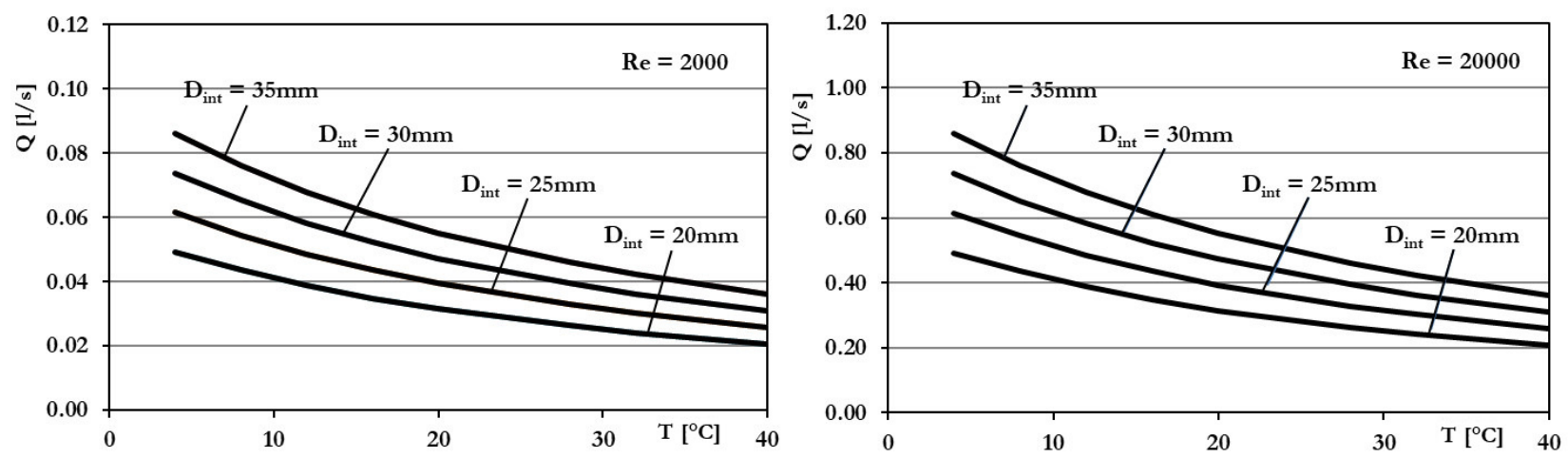

Figure 4 Flow rate vs. temperature of the thermal probe circuit, for two Reynolds numbers and some probe diameters.

Figure 4 shows the flow rate in the probe circuit when the temperature of the fluid (water) and the diameter of the probe tube vary. On the left, a description of the situation at the limit of laminar flow (the Reynolds number, Re, is 2000). The maximum flow rate, $\sim 0.10 \mathrm{l} / \mathrm{s}$, is obtained at low temperatures with the maximum diameter considered of $35 \mathrm{~mm}$. This flow rate is easily achieved with the designed power of the circuit. On the right, a description of the case in which the Re in the probe circuit reaches 20000 . Considering the maximum diameter, at temperatures slightly above $0^{\circ} \mathrm{C}$ the flow rate is $0.9 \mathrm{l} / \mathrm{s}$, close to the maximum value obtainable from the system under the conditions described previously. These Re values are close to the maximums technologically used. The limits of the filtration fluid velocity in the porous medium are obtained as a function of the maximum generable rate of flow (between 0.25 $1 / \mathrm{s}$ and $1 \mathrm{l} / \mathrm{s})$, the maximum piezometric gradient $(\sim 0.2 \mathrm{~m} / \mathrm{m})$ and the filtration area $\left(\sim 1 \mathrm{~m}^{2}\right)$. The velocity ranges between $2.7 \times 10^{-4} \mathrm{~m} / \mathrm{s}$ and $1.21 \times 10^{-3} \mathrm{~m} / \mathrm{s}$. In the case of a Darcy flow, the hydraulic conductivity varies between $1.4 \times 10^{-3} \mathrm{~m} / \mathrm{s}$ and $5.5 \times 10^{-3} \mathrm{~m} / \mathrm{s}$, which is the case of clean sand, sand and gravel. 


\section{Temperature sensors}

The temperature measurement was carried out by means of Resistance Temperature Detectors (RTDs). The thermocouples were excluded due to their insufficient accuracy and the thermistors were excluded because of their highly non-linear calibration curves. In particular, PT100 RTDs with three-wire connections were used. They have an adequate sensitivity (less than $0.1^{\circ} \mathrm{C}$ ) and a calibration curve very close to linearity, although their response time is not particularly short, especially if the sensor is protected by a copper capsule saturated with thermally conductive resin. The experimental tests performed on the bare probes showed a behavior consistent with the manufacturer data (variation of $90 \%$ of the temperature jump in a time of $0.3 \mathrm{~s}$ ), while the probes protected with copper capsules of different shapes and lengths presented a response time of approximately $10 \mathrm{~s}$. To reduce the response time up to 2-3 seconds, the protection was achieved by means of a water-permeable metal sock. This response time is considered compatible with the temperature variation times that occur in the physical model.

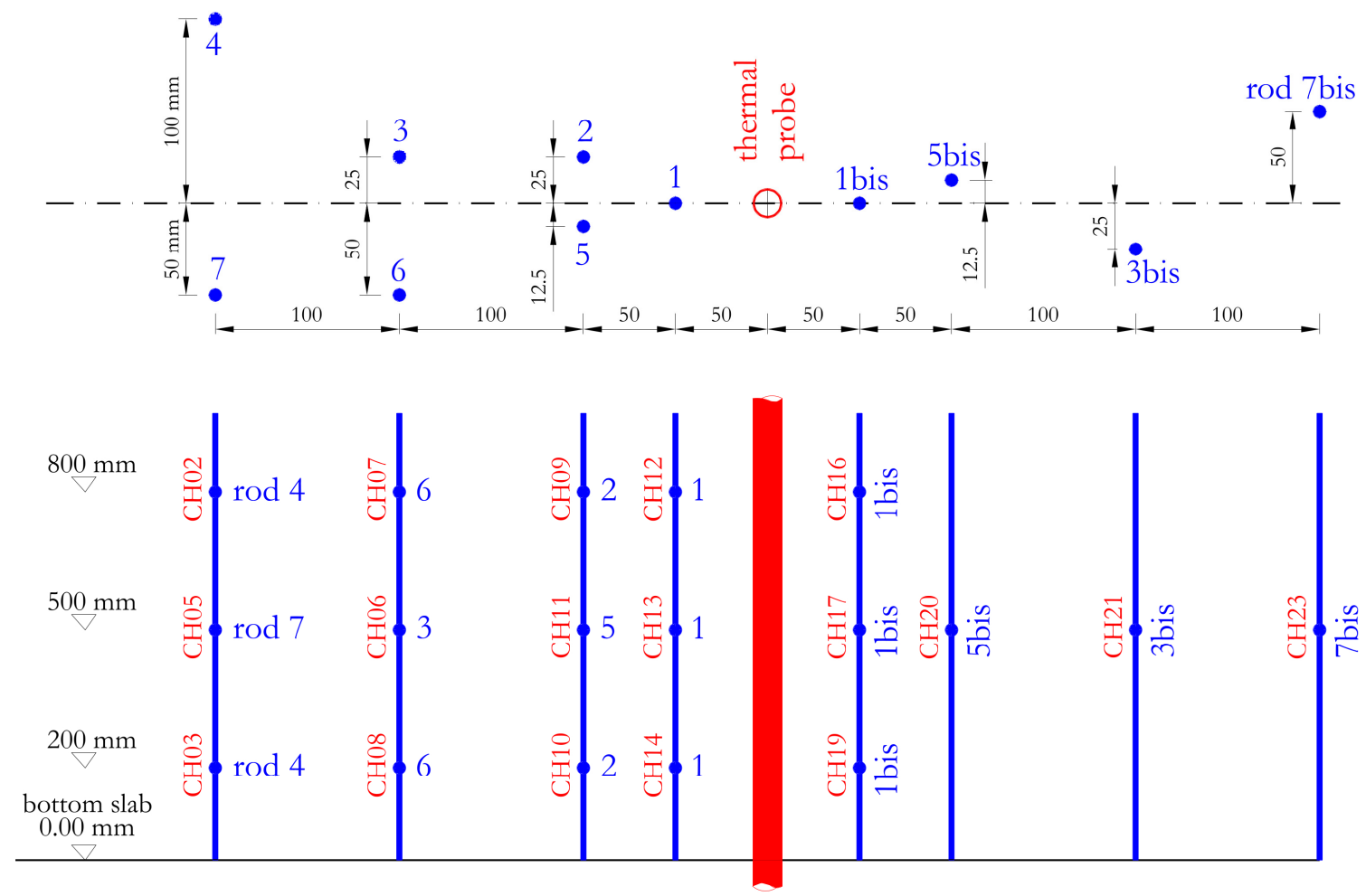

Figure 5 Above: planimetric position of the rods supporting the RTDs inside the control volume; below: rod and elevation with respect to the bottom of the control volume of each RTD.

In the tests described here, the RTDs are used to measure the temperature of: the fluid in the copper probe (input and output fluid temperature); the filtration fluid entering the control volume; the environment; 18 positions within the control volume. The RTDs are arranged within the control volume on the basis of a preliminary finite element analysis carried out by means of Comsol Multiphysics, taking into account the test configurations in the absence and in the presence of filtration water. The planimetric and altimetric layout is shown in Figure 5. Wooden vertical rods with $6 \mathrm{~mm}$ diameter are placed in the positions indicated in the plan. The RTDs are placed on these rods, in the points shown in the Figure 5, and facing the copper probe. The rods are placed at different distances from the copper probe and are arranged mainly in the direction of the flow in the tests with filtration motion, in greater number downstream of the probe (rods $1,2,3,4,5,6,7)$ but also upstream (rods 1 bis, 5bis, 3bis, 7bis). The 
arrangement of the downstream rods is aimed at characterizing the temperature plume which is formed in a limitedwidth area downstream of the probe in the case of simulation of the groundwater flow. In the absence of groundwater flow, the temperature distribution should assume an axis-symmetrical behavior with respect to the thermal probe. The limits of this hypothesis are evaluated by means of the RTDs placed on the rods located upstream, in a symmetrical position with respect to the rods arranged downstream. The acquisition system has been designed to serve a maximum of 42 analogic channels.

\section{THE FIRST TESTS}

The assessment of the behavior of the experimental device was carried out by filling the control volume with different materials, leading to 5 experimental configurations: (i) water; (ii) moving water; (iii) dry granular material; (iv) saturated granular material; and (v) saturated granular material with moving water. The experimental results obtained in configurations (iv) and (v) are shown here. Figure 6 shows two images (on the left and in the middle) of the device in the configuration of the tests with saturated granular material. The image on the right shows the rods used as a support for the RTDs distributed inside the control volume. The volume was filled with "Risetta del Brenta" (literally, small rice from the Brenta river), a sandy material obtained from the riverbed of the Brenta river near Padua. The deposition of the granular material inside the model was obtained by letting the granular material fall into still water. The measurement of the extracted water volume $\left(0.505 \mathrm{~m}^{3}\right)$ and of the mass of the material put into the model $(1365.17 \mathrm{~kg})$ allowed the estimate of the average density of the solid component, $2700 \mathrm{~kg} / \mathrm{m}^{3}$, and of its porosity, 0.43 (Figure 6).

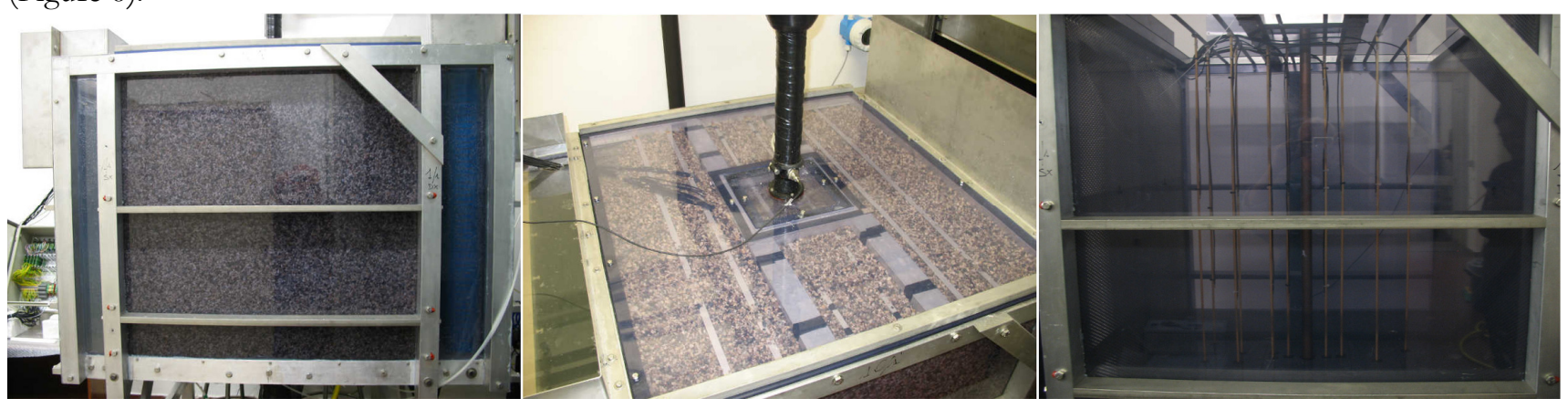

Figure 6 On the left and in the middle, two images of the experimental device filled with granular material saturated with water. On the right, a view of the inside of the control volume with the RTDs supporting rods. .

Figure 7 shows the experimental data acquired during the test carried out without filtration motion. The average input and output fluid temperatures at the copper probe are $43.0^{\circ} \mathrm{C}$ (standard deviation, $\mathrm{SD}$, of $0.4^{\circ} \mathrm{C}$ ) and $42.4^{\circ} \mathrm{C}\left(0.4^{\circ} \mathrm{C} \mathrm{SD}\right)$ respectively. The average temperature difference between the two sensors is $0.57^{\circ} \mathrm{C}\left(0.06^{\circ} \mathrm{C} \mathrm{SD}\right)$. The average room temperature is $18.7^{\circ} \mathrm{C}\left(0.3^{\circ} \mathrm{C} \mathrm{SD}\right)$. The flow rate of the fluid in the copper probe is $10.12 \mathrm{l} / \mathrm{min}$ $(0.03 \mathrm{l} / \mathrm{min} \mathrm{SD})$. Figure 7 , on the left, shows that permanent boundary conditions of the input temperature, as well as permanent flow rate in the thermal probe and permanent room temperature can be achieved with the proposed device. The difference between input and output temperatures of the thermal probe is significant compared to the accuracy of the RTDs used $\left(\sim 0.025^{\circ} \mathrm{C}\right)$ and leads, through the application of the energy balance equation, to an estimate of the probe power per unit length of $400 \mathrm{~W} / \mathrm{m}$, slightly decreasing over time (an order of magnitude higher than the peak technological values). Figure 7 , on the right, shows the temperature trends in symmetrical positions within the control volume (CH12-CH16; CH13-CH17; CH14-CH19; CH11-CH20; CH06-CH21; CH05-CH23). The temperature pattern is almost symmetric, as expected. The arrival times of the thermal wave are substantially concordant. The differences in temperature detected could be mainly related to a possible lack of homogeneity of the granular material and to the non-precise symmetry in the positioning of the probes within the control volume, 
especially near the thermal probe where the temperature gradients are greater.
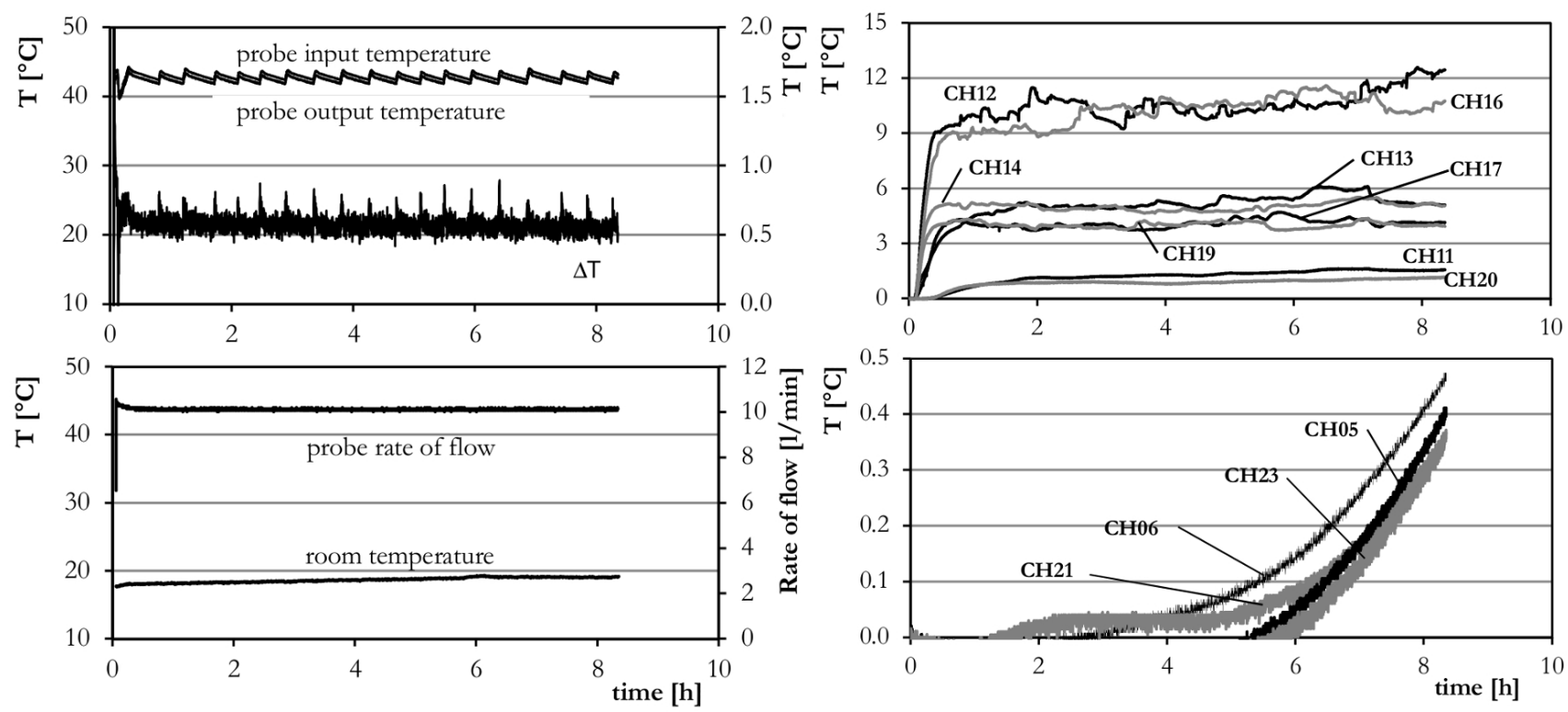

Figure 7 Experimental data acquired during the test with granular material saturated with still water. On the left, the input and the output temperature of the thermal probe fluid, its discharge and the room temperature. On the right, the temperature measured by some RTDs inside the control volume in a symmetrical position to the thermal probe.
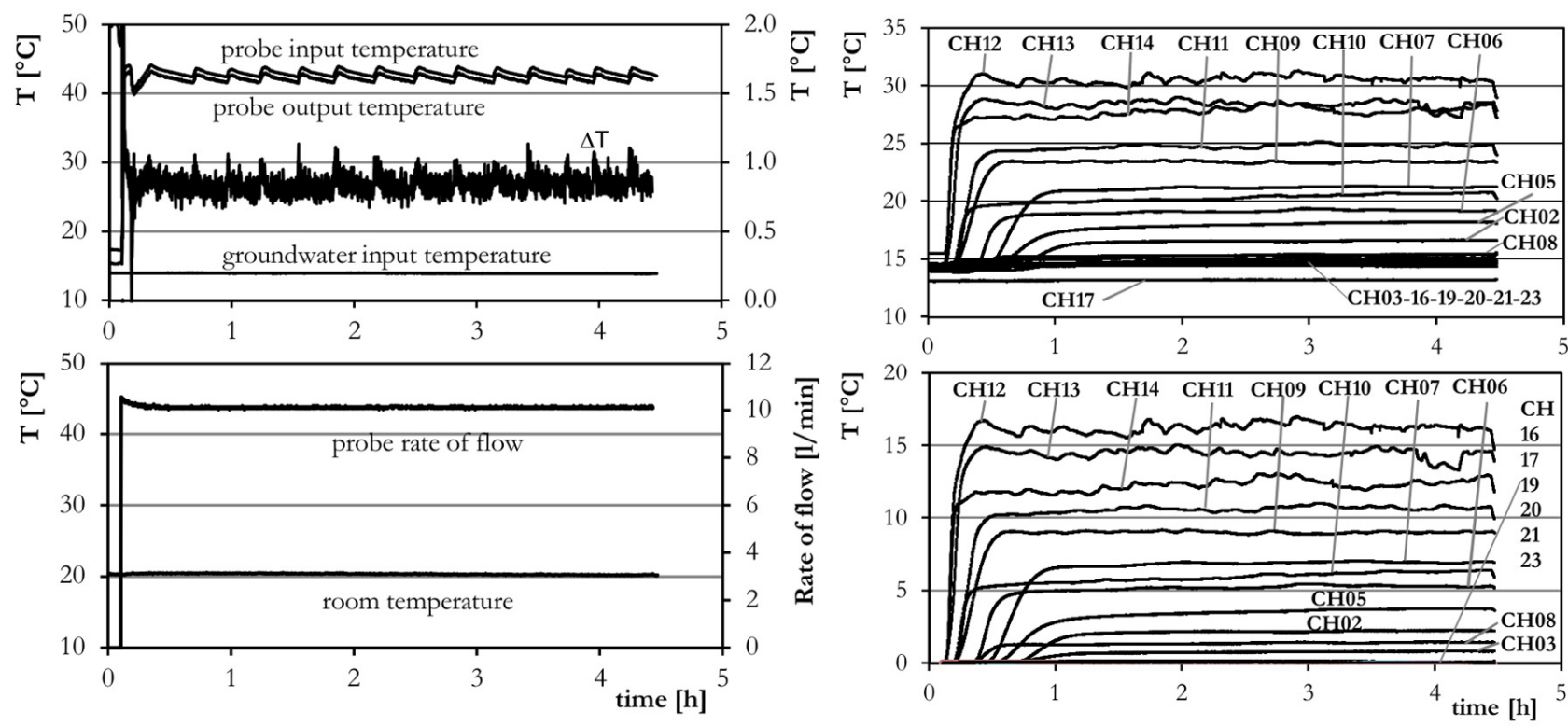

Figure 8 Experimental data acquired during the test with granular material saturated with filtration flow. On the left, the input and the output temperature of the thermal probe fluid, the temperature of the filtration fluid, the discharge of the probe fluid and the room temperature. On the right, above, the temperature measured by the RTDs inside the control volume; below, the temperature values with respect to the initial values.

Figure 8 shows the experimental data acquired during the test carried out with filtration motion. The average input and output fluid temperatures at the copper probe are respectively $43.0^{\circ} \mathrm{C}\left(0.4^{\circ} \mathrm{C} \mathrm{SD}\right)$ and $42.2^{\circ} \mathrm{C}\left(0.4^{\circ} \mathrm{C} \mathrm{SD}\right)$. 
The average temperature difference between the two sensors is $0.84^{\circ} \mathrm{C}\left(0.07^{\circ} \mathrm{C} \mathrm{SD}\right)$, much higher than in the previous test. The average room temperature is $18.7^{\circ} \mathrm{C}\left(0.3^{\circ} \mathrm{C} \mathrm{SD}\right)$. The flow rate of the fluid in the copper pipe is $10.12 \mathrm{l} / \mathrm{min}$ $(0.031 / \mathrm{min} \mathrm{SD})$. The velocity of the fluid in the copper probe is $0.274 \mathrm{~m} / \mathrm{s}$ and the corresponding Reynolds number, at the temperature of $40^{\circ} \mathrm{C}$, is 11654 . This implies that the flow regime in the probe is a transition between laminar and turbulent motion. The filtration flow rate, obtained by mean of the volumetric method, is $4.721 \mathrm{l} / \mathrm{min}(0.017$ $1 / \mathrm{min} \mathrm{SD}$ ). The average filtration velocity is $8 \cdot 4 \cdot 10^{-5} \mathrm{~m} / \mathrm{s}$. Based on the measurements made with piezometers, the piezometric drop is $0.0166 \mathrm{~m} / \mathrm{m}$. In the reasonable hypothesis of validity of Darcy's law, a hydraulic conductivity of $5.06 \cdot 10^{-3} \mathrm{~m} / \mathrm{s}$ is obtained, which agrees with data from literature. The average temperature of the input filtration fluid is $13.92^{\circ} \mathrm{C}\left(0.03^{\circ} \mathrm{C} \mathrm{SD}\right)$. In this case the estimate of the probe power per unit length is $595 \mathrm{~W} / \mathrm{m}$. Figure 8 , on the left, shows the same data of the previous test plus the temperature of the filtration fluid: the boundary conditions are stable. On the right, the figure above shows the temperature trends measured by the RTDs inside the control volume; below, the same trends referring to the initial local temperature values. It should be noted that the temperatures at the beginning of the test are not equal in the various positions within the control volume. This should be due mainly to the difficulty of obtaining homogeneous initial conditions within the volume of control because of the significant size of the physical model and the size of the environment in which it is located, and, in a minor part, to a possible alteration of the temperature sensor calibration curves. In this test temperature variations are significant only downstream from the thermal probe: all RTDs located upstream (Figure 5) do not experience any temperature modification.

\section{CONCLUSIONS}

A new experimental apparatus was designed and constructed in order to study the physical processes that take place when a thermal probe exchanges energy with the surrounding environment. This paper presents the apparatus and two tests performed in order to evaluate its capability to fulfil design requirements.

In the tests carried out with saturated granular material, at rest or moving, the amplitudes of the temperature variations induced in the fluid inside the thermal probe were significantly higher than the sensitivity of the RTDs and an accurate analysis of the energy exchange between the thermal probe and the surrounding environment was possible, together with the description of the temperature field in the control volume over time. In the tests carried out with still water, the expected axial symmetry of the temperature pattern emerged clearly.

The thermal probe circuit is suitable for the purposes of the tests. A highly stable fluid discharge was obtained. The stability of the temperature sensors appeared to be adequate. The results also suggested some improvements of the system for further experimental stages (e.g., methods of RTDs positioning in the control volume more accurate could be used).

It is reasonable to assume that the experimental apparatus here proposed is a powerful tool for the analysis of the complex phenomena under study. Its use can be aimed at understanding the role of the different physical parameters involved in the phenomena and at characterizing different materials and different technical solutions for heat exchangers. Moreover, the experimental data can be used in the construction and calibration process of numerical models.

\section{ACKNOWLEDGMENTS}

The research has been funded in the framework of the research project "GEOTERM (Thermal Geo-Exchange in the Province of Trento)", REET-FBK, Trento, Italy. Moreover, the authors wish to thank Tecnopenta s.r.l., Teolo (Padua) for their aid in the development of the experimental device and Simonetta Cola, head of the Geotechnical Engineering Laboratory, DICEA, University of Padua, where the experimental set up was installed. 


\section{REFERENCES}

Al-Khoury, R., Bonnier, P.G. and R.B. Brinkgreve. 2005. Efficient finite element formulation for geothermal heating system. Part I: Steady state. International Journal for Numerical Methods in Engineering 63: 988-1013.

Al-Khoury, R., and P.G. Bonnier. 2005. Efficient finite element formulation for geothermal heating system. Part II: Transient. International Journal for Numerical Methods in Engineering 67: 725-745.

Carlini,M., Castellucci, S., Allegrini, E. and A. Tucci. 2012. Down-Hole Heat Exchangers: Modelling of a Low-Enthalpy Geothermal System for District Heating. Mathematical Problems in Engineering 2012(845192): 1-11.

Cimmino, M., and M. Bernier. 2015. Experimental determination of the g-functions of a small-scale geothermal borehole. Geothermics 56: 60-71.

Green, D.L. 2014. Modelling Geomorphic Systems: Scaled Physical Models. Section 5.3 in Cook, S.J., Clarke, L.E. and J.M. Nield (Eds.) Geomorphological Techniques. London: British Society for Geomorphology

IEA. 2011. Technology Roadmap - Geothermal Heat and Power. Technical report. Paris: International Energy Agency.

Li, W., Li, X., Peng, Y., Wang, Y., and J. Tu. 2018. Experimental and numerical investigations on heat transfer in stratified subsurface materials. Applied Thermal Engineering 135: 228-237.

Philippe, M., Bernier, M. and D. Marchio. 2009. Validity ranges of three analytical solutions to heat transfer in the vicinity of single boreholes. Geothermics 38: 407-413.

Scotton, P. 2017. Un apparato sperimentale per lo studio dello scambio energetico tra sottosuolo e sonda geotermica, Project GEOTERM (Geoscambio nella Provincia Autonoma di Trento). Trento: Reet-FBK.

Sutton, M, Nutter, D., and R. Couvillion. 2003. A ground resistance for vertical bore heat exchangers with groundwater flow. Journal of Energy Resources Technology 125: 183-189.

Volkov, A.V., Ryzhenkov, A.V., Kurshakov, A.V., Grigoriev, S.V. and V.V. Bekker. 2017. Physical Modelling of Temperature's Potential Decrease for Near-Wellbore Rocks during Extraction of Thermal Energy. International Journal of Applied Engineering Research Volume 12(17): 6570-6575.

Wang, Y., Gao, Q., Zhu, X., Yu, M., and X., Zhao. 2013. Experimental study on interaction between soil and ground heat exchange pipe at low temperature. Applied Thermal Engineering 60(1-2): 137-144. 